\title{
Extending Electromagnetic Treatment to Infectious Diseases including the Ebola Virus
}

\author{
Edgar E. Escultura \\ Research Professor (Honorary), GVP-Professor V. Lakshmikantham Institute for Advanced Studies GVP College \\ of Engineering, JNT University Kakinada, Vishakhapatnam, India \\ Email: escultur36@gmail.com
}

Received 20 October 2014; accepted 10 April 2015; published 14 April 2015

Copyright (C) 2015 by author and Scientific Research Publishing Inc.

This work is licensed under the Creative Commons Attribution International License (CC BY). http://creativecommons.org/licenses/by/4.0/

(c) (i) Open Access

\begin{abstract}
A blueprint for research and development for design and development of suitable electromagnetic treatment of genetic diseases (Escultura, Journal of Biomaterials and Nanobiotechnology, 3(2), 2012) known as genetic sterilization is applied to infectious diseases including viruses. It is based on the Grand Unified Theory (Escultura, Nonlinear Analysis, A-Series, 69(3), 2008) and the Unified Theory of Evolution (Escultura, The Journal of the Science of Healing Outcomes, 2015). Since every physical characteristic of living thing is determined by a gene, so is the undesirable symptom of a disease. For virus, its undesirable symptom is determined principally by the wavelength of the natural vibration of its gene as sequence of base materials which is equal to its measure as a segment of a double helix. Radiation with wavelength equal to this measure will resonate with it. Raising its intensity suitably will sterilize the gene, stop its spread and render it harmless to the host; then the disease is treated without harm to normal cells. Another treatment is establishing resonance with radiation of wavelength equal to the measure of the virus (protein strand with the gene) across; raising its intensity suitably will burn the virus without side effect (since it is much smaller than normal cells).
\end{abstract}

\section{Keywords}

Basic Primum, Genetic Activation and Sterilization, Resonance and Superposition Law, Superstring

\section{Introduction}

This paper was inspired by the challenge posed by the ebola virus. In 2012, the author published, Electromagnetic Treatment of Genetic Diseases [1], a practical application of the Grand Unified Theory (GUT) [2] and the 
Unified Theory of Evolution (UTE) [3]. There are two types of this treatment. One is the introduction of a neutralizer gene that removes the undesirable symptom of the genetic disease [3] through resonance and superposition [4] [5] and treats the diseases effectively; it has been quite successful for cystic fibrosis and can be extended to other genetic diseases for treatment except cancer [1] [3] [6]. The other treatment involves radiation that resonates with the determinant gene of the disease. By raising its intensity sufficiently, its gene is sterilized or destroyed without injury to normal cells. The treatment was originally aimed at the common genetic diseases: cancer, systemic lupos erythematosus, diabetes, muscular dystrophy and mental disorder. However, since physical characteristics and behavior of living things including plants, organisms and viruses are determined by their genes [3], this treatment applies to diseases caused by them. It requires only sterilization of the gene responsible for the harmful symptom. Although we focus on the virus, this paper opens up more possibilities for resonance with and treatment of infections.

\section{The New Methodology}

The methodology here is qualitative mathematics and modeling (QMAM) introduced in and the main contribution of [7]. Qualitative mathematics is the complement of computation and measurement and the qualitative model of rational thought [8]; it includes the following activity:

Making conclusions, visualizing, abstracting, thought experimenting, learning, creating concepts for building mathematical space, intuition, imagination, trial and error to sift out what is appropriate, negating what is known to gain insights into the unknown, altering premises to draw out new conclusions, thinking backwards, finding basic premises for a mathematical space and the search for laws of nature.

Qualitative modeling explains natural phenomena (appearances of nature) and how nature (our universe) works in terms of natural laws its main tool qualitative mathematics [8]. This new methodology was applied to physics for the first time to solve the gravitational n-body problem [9]. The solution required the discovery of the superstring, fundamental building block of matter [10], which, in turn, required the discovery of the initial natural laws of GUT. In contrast, the traditional methodology of physics called quantitative modeling (formerly called mathematical modeling) describes the appearances of nature mathematically, e.g., by numbers and equations, its main tools computation and measurement that failed to resolve long-standing problems of mathematics, e.g., Fermat's Conjecture (also known as Fermat's last theorem or FLT [11]). Quantitative modeling failed to solve long-standing problems of physics, e.g., the gravitational and turbulence problems, and answer fundamental questions such as what the fundamental building block of matter and the structure of the electron are. Thus, the need for this new methodology to advance physics and establish it as the only truly basic science since it is central to the natural sciences and their applications. In effect, QMAM shifts the subject matter of physics from the appearances of nature to nature itself and how it works and lifts traditional to the new physics articulated by GUT [8]. In fact, it is the only methodology capable of creating or formulating physical theory and the appropriate mathematics for it [12].

True to its name, GUT encompasses and unifies the natural sciences on the natural laws the superstring being the common thread. GUT has theoretical applications in biology [3] [13] and physical psychology [14]-[16] and practical applications in engineering [17] [18], medicine [1] [6], geological, atmospheric and climate sciences and oceanography [19]-[22], mathematics-science education [23] [24] and sustainable development of the Third World [25] [26].

\section{The Grand Unified Theory}

We summarize both physical theories GUT and UTE for applications to the treatment of genetic and infectious diseases with focus on the latter.

\subsection{Quantum Gravity}

Quantum gravity, one of the three pillars of GUT, is the science of physical systems at the atomic scale or less, an extension of quantum physics to dark matter (the others are thermodynamics in the broad sense and macro gravity).

\subsection{Physical Concepts and Natural Laws}

Mathematical concept is created by thought [15], physical concept comes from sensation in the creative-integra- 
tive region (CIR) of the cortex induced by external signals from event or object in nature, its referent, through the sense organs or derived from natural laws [15] [16]. We prove the existence of the superstring.

What appears empty Cosmos other than cosmological bodies is actually dark matter, i.e., matter not observable by the medium of light, one of the two fundamental states of matter known only by its impact on ordinary or visible matter, the other fundamental state. Its existence follows from the steady appearance of matter in the Cosmos at the rate of one star per minute [27]-[29] and this fundamental natural law.

\subsection{Energy Conservation}

In any physical system and its interaction, the sum of kinetic (visible) and latent (dark) energy is constant, gain of energy is maximal and loss of energy is minimal.

What does dark matter consist of? Our answer: the superstring. The full derivation of its structure (nested generalized physical fractal [30] [31] that insures its indestructibility otherwise our universe would have collapsed long ago which did not [2]), properties and behavior is in [10]. That it is unique like the electron follows from its being the fundamental building block of matter.

Energy Conservation expresses itself in many ways and forms stated in the next natural law.

\subsection{Energy Conservation Equivalence}

Energy conservation has many expressions or forms: order, symmetry, economy, least action, optimality, efficiency, stability, self-similarity (nested fractal), coherence, resonance, quantization, synchronization, smoothness, uniformity, motion-symmetry balance, non-redundancy, non-extravagance, evolution to infinitesimal configuration, helical and related configuration such as circular, spiral and sinusoidal and, in biology, genetic encoding of characteristics, reproduction and order in diversity and complexity and configuration that provides optimal capability. (We call each component here physical principle).

Energy is motion of matter; therefore, matter and energy are never separate and anything that has energy, e.g., photon, has mass; conversely, anything massless is not matter. Wave is suitably synchronized vibration of the medium [10]. Basic cosmic or electromagnetic wave is generated by the natural vibration of atomic nucleus propagated across dark matter via Resonance [4] [5] [10]; dark matter fills up the Cosmos. The only external physical systems that interact with the superstring are electromagnetic and seismic wave [10] [21]. Flux is motion of matter with identifiable direction at each point, e.g., water current. Turbulence is coherent flux, e.g., hurricane, wave. Chaos is mixture of order none of which is identifiable. For example, chaos occurs at the onset of turbulence as transitional phase towards it [20]. Normal nuclear vibration is due to the impact of electromagnetic and seismic waves [11] [21] coming from all directions its characteristics determined by the nuclear structure in accordance with this natural law (inspired by Engel’s Dialectics of Nature [32]).

\subsection{Internal-External Factor Dichotomy Law}

The interaction, dynamics and physical characteristics of a physical system is shaped by internal and external factors; in general the internal is principal over the external and the latter works through the former.

The next law inspired by a high school experiment many years ago is central to the study of turbulence and derivation of the structure and configuration of the superstring as nested generalized physical fractal, the only structure that makes it indestructible [10] [30].

\subsection{Flux-Low-Pressure Complementarity}

Low pressure sucks matter around it and the initial rush of matter towards region of low pressure stabilizes into local turbulence, e.g., hurricane; conversely, coherent flux induces low pressure around it.

The proof of the existence of the superstring and derivation of its structure, properties and behavior is in [10]. We state the result as the next natural law.

\subsection{Existence of the Fundamental Building Block of Matter and Its Nested Generalized Fractal Structure}

The fundamental building block of dark matter is the non-agitated superstring. It is a circular helical loop and nested fractal sequence of superstrings or toroidal fluxes, with itself as first term; each toroidal flux in the se- 
quence is a superstring having toroidal flux, a superstring, traveling at $7\left(10^{22}\right) \mathrm{cm} / \mathrm{sec}$ along its cycles, etc.; each superstring except the first, is contained in and self-similar to the preceding term in structure, behavior and properties.

This speed of $7\left(10^{22}\right) \mathrm{cm} / \mathrm{sec}$ was measured as the linear speed of the gravitational flux of the proton [33] but by the synchronization and quantization principles this is a constant of nature, e.g., the speed of electric current.

The first term of the nested fractal superstring is a circular helix that forms a loop (Figure 1) containing a superstring traveling through its loop at $7\left(10^{22}\right) \mathrm{cm} / \mathrm{sec}$ called toroidal flux. (For the full derivation, see [10]).

The superstring is non-agitated if cycle length (CL) $<10^{-16} \mathrm{~m}$, semi agitated if $10^{-16}<\mathrm{CL}<10^{-14} \mathrm{~m}$, agitated if segment has CL $>10^{-14} \mathrm{~m}$. Steady shrinking due to Energy Conservation makes the semi-agitated phase almost instantaneous. Thus, non-agitated superstrings comprise almost all of dark matter.

We also identify the superstring with its first term (Figure 1) since it is this term that interacts with other physical systems. The nested fractal sequence of superstrings (toroidal fluxes) that comprises the superstring has no last element and that is what makes it indestructible. For, when cosmic wave hits a superstring at most finite terms of its fractal sequence of superstrings break leaving the tail intact and nested fractal sequence of superstrings, i.e., a superstring. It follows that the Universe of dark matter has no beginning and no end (timeless) [34]. Moreover, by Flux-Low-Pressure Complementarity, it has no boundary, i.e., unbounded and infinite and our universe is a finite local bubble in it among other universes as shown by galaxies traversing our universe from outside as well as collision of galaxies coming from different directions [35] [36]).

When suitable electromagnetic wave hits a non-agitated superstring it converts to a semi-agitated superstring but when it hits a semi-agitated superstring a pair of mutually exclusive events occurs: a) the first term of its fractal superstring bulges to retain the toroidal flux speed despite the energy imparted on it, by Energy Conservation, turning it into an agitated superstring called primum, unit of visible matter, its toroidal flux non-agitated, or b) the first term breaks, its toroidal flux, a superstring, remaining non-agitated (dark). The next law articulates our findings.

\subsection{Dark-to-Visible-Matter Conversion}

When suitable electromagnetic wave hits a semi-agitated superstring one of these occurs: a) the outer superstring breaks, its toroidal flux remaining non-agitated superstring; b) a segment bulges into a primum, an

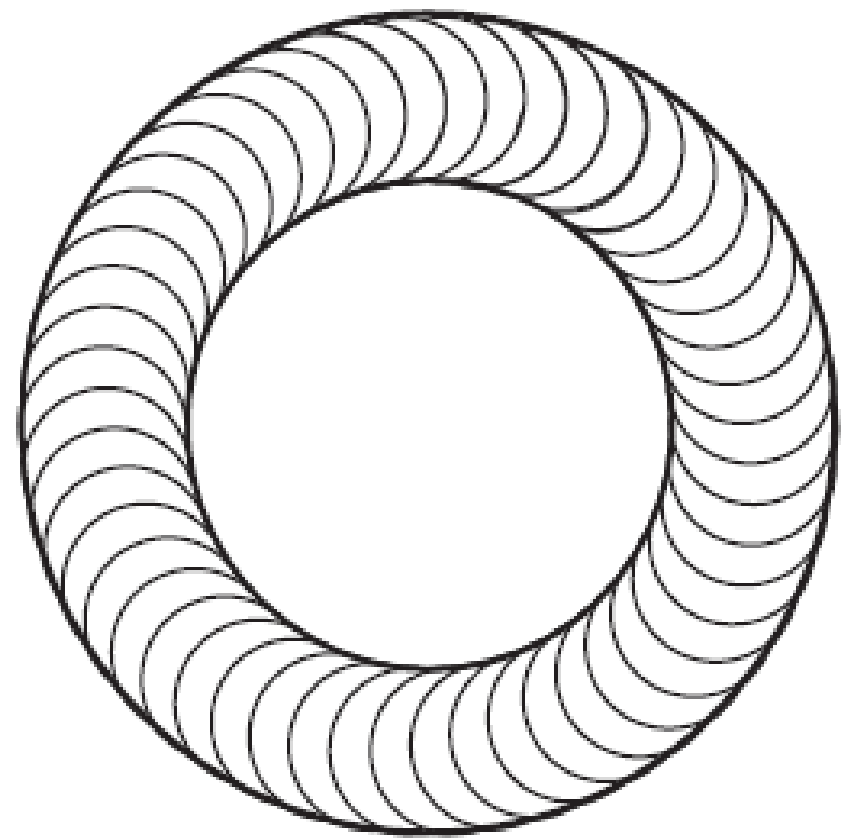

Figure 1. An artist's conception of a semi-agitated superstring. A non-agitated superstring, its toroidal flux, has a similar nested fractal structure as itself and travels through its helical cycles at the speed of $7 \times 10^{22} \mathrm{~cm} / \mathrm{sec}$ [4]. The helix winds around the torus rapidly its cycles infinitesimally close. 
agitated superstring and a unit of visible matter.

The next law governs flux interactions.

\subsection{Flux Compatibility*}

Fluxes of the same direction attract but fluxes of opposite directions repel.

\subsection{The Basic Prima}

What is a primum? When the toroidal flux along its cycles is hit by cosmic waves coming from all directions it is thrown into erratic motion and bounces with other superstrings turning it into a spike [10] with the centroid traveling through the cycles at $7\left(10^{22}\right) \mathrm{cm} / \mathrm{sec}$. It pulls the superstrings around the primum into its induced vortex flux of superstrings with axis coinciding with its axis inside the cylindrical eye making it a magnet, its polarity in accordance with the right hand rule of electromagnetism (Figure 2). The induced vortex flux is counterclockwise for a positive primum, by convention, negative otherwise. The plane through the apex of its profile and normal to its axis is the equatorial plane its intersection with the rim of its vortex the equator.

The induced vortex flux is measured as charge, its unit the electron's charge: $-1\left(1.6\left(10^{-19}\right)\right.$ coulombs). The electron, +quark, -quark, charges $-1,+2 / 3,-1 / 3$, respectively [37], are basic prima since they comprise the atom. Seismic waves generated at the interface of turbulence in the spinning cores of cosmological vortices [34] in the Cosmos convert dark to visible matter around them at one star per minute [27]-[29].

The primum's toroidal and induced vortex fluxes and natural vibration of dark matter endow dark matter huge latent energy partially convertible to kinetic (visible) energy. The latent energy density of dark matter: $10^{26}$ joules/cubic ft. (de Broglie [38]) or $8\left(10^{8}\right)$ volts/cm (Seike Jr [38]), the equivalent of $18 \mathrm{~kg} / \mathrm{cu}$ meter (Gerlovin [37], relativistic conversion).

The next natural law is special form of Flux Compatibility ${ }^{*}$ that applies to vortex fluxes directly.

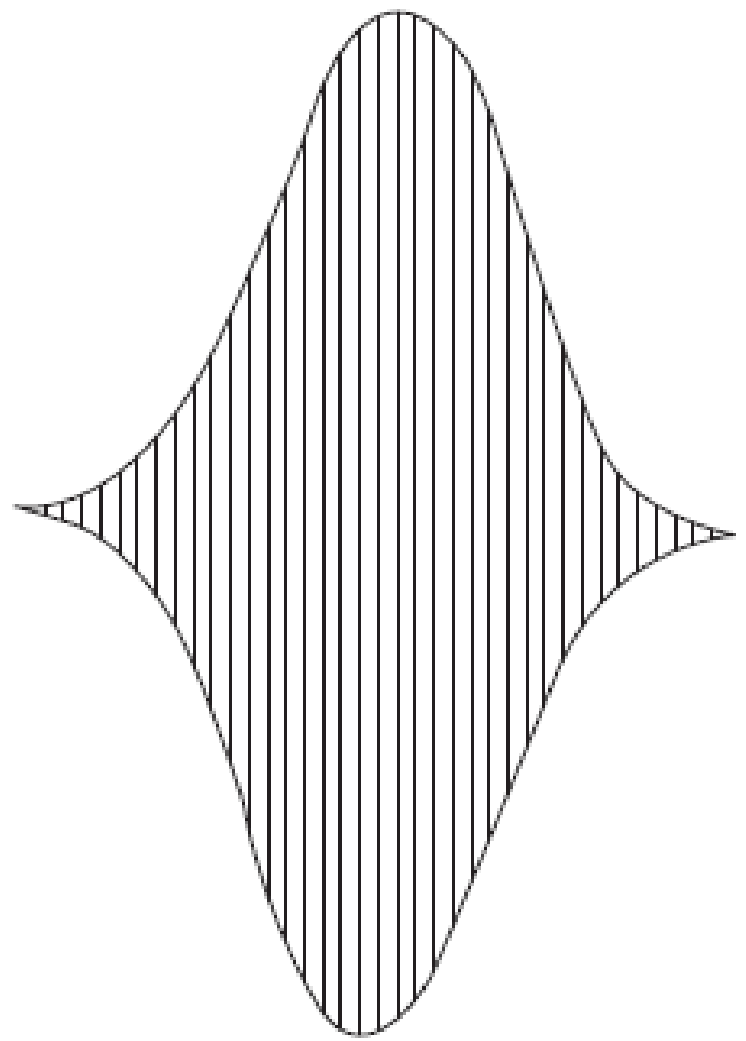

Figure 2. A simple primum, bulged segment of semi-agitated superstring and a magnet with induced vortex flux hidden. Its polarity conforms to the right-hand-rule of electromagnetism: when the index finger points to the direction of its toroidal flux, the thumb points to the $\mathrm{N}$-pole. 


\subsection{Flux Compatibility}

Two prima of opposite toroidal flux spins attract at their induced vortex flux rims but repel at their poles; otherwise, they repel at their rims but attract at their poles. Two prima of same toroidal flux spins connect at their rims only through a primum of opposite toroidal flux spins between them.

\subsection{Primal Interaction}

Primal interaction is governed by Flux Compatibility and Flux-Low-Pressure Complementarity.

The proton consists of two +quarks joined by a -quark at their rims, by Flux Compatibility (Figure 3(a)), their axis coplanar (Energy Conservation), its charge: $2 / 3-1 / 3+2 / 3=1$. Since simple primum is charged, the neutral neutrino is coupled prima of numerically equal but opposite charges, say, $+q$ and $-q$, its charge $+q+-q$ $=0$, neutral.

The electron attaches to both +quarks beside the - quark but away from the negative quark of the proton, their centers viewed from the north-pole forming a quadrilateral. Their coherent fluxes in the interior suck only light neutral primum, the neutrino, to form the neutron (Figure 3(b)). This is the neutron, its charge: $+2 / 3-1 / 3+2 / 3$ $-1+0=0$, neutral. Since the neutron's, proton's and electron's masses are, respectively, 1.0087, and $5.486 \times$ $10^{-8} \mathrm{amu}$, the neutrino's mass is $\eta=8.5\left(10^{-8}\right)$ amu or 1.55 times the electron's mass [39].

The protons form the nucleus of the atom first (Figure 4). The vortex fluxes (charges) add up around it to form the vortex flux (charge) of the atom. They are joined together pairwise by -quarks (their arrangement discussed in [10]). Since the nucleus is fractal the electromagnetic wave it generates is fractal and endowed with huge energy.

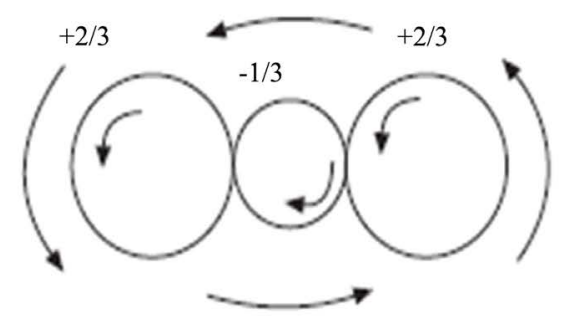

(a)

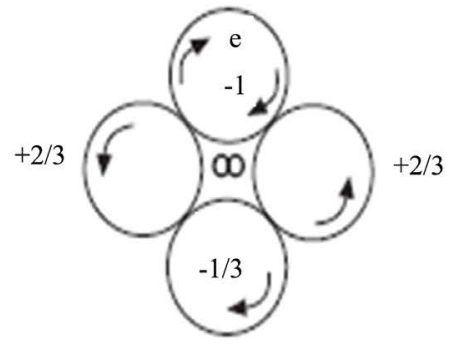

(b)

Figure 3. The proton: two +quarks joined by a -quark at their vortex fluxes’ rim by flux compatibility (a). The neutron consisting of a proton, electron and neutrino (b); the neutrino is represented by a figure 8 since it is a coupled primum of opposite but numerically equal charge joined likewise at their fluxes’ rims.

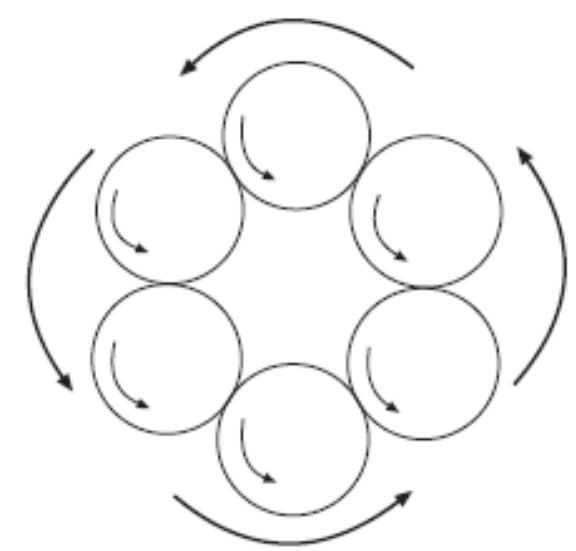

Figure 4. Light nucleus viewed from the north-pole. The -quarks that join the protons are not shown as the -quarks is $1 / 400$ as massive as the proton. In reality the protons are joined by the -quarks at rims of their vortex fluxes, by Energy Conservation. The neutrons are not indicated as they do not contribute to the vortex flux of the atom; moreover, they are neutral (no net vortex fluxes around them). 
The nucleus is a magnet of positive polarity, the vortex flux around it its magnetic field. Viewed from the north-pole the vortex flux of the atom spins counterclockwise.

Being negatively charged the electrons are attracted to the vortex flux away from the eye but being light, they are swept into orbit by it. By centrifugal force, the most energetic orbital electrons are closest to the equatorial plane near the equator; they form the outermost orbital shells; the least energetic cluster near the poles and form the lowest orbital shells. Moreover, the eye sucks non-agitated superstrings that accumulate steadily in the nucleus as mini black hole [10], the main source of nuclear energy released in nuclear fission. In fact, every simple charged primum sucks non-agitated superstrings and accumulates them as mini black hole in the eye. The Higgs bosom detected in CERN [40] when two protons at great speed collided were mini black holes, a maximum of six of them that could have been detected since there was a total of six simple prima between the two colliding protons. Therefore, the Higgs boson is not the fundamental building block of matter but a mini black hole. In fact, the only evidence of its existence was its destruction at CERN [40].

(The figures in this section are taken from the book, Scientific Natural Philosophy [41].)

\section{The Unified Theory of Evolution}

We identify natural laws that apply to biological systems and call them biological laws.

\subsection{Some Biological Laws}

Since in biology we deal with the tiny superstrings in large numbers, sometimes at great speed, processes, motion, etc., interactions are stochastic (uncertainty of small and large numbers [42] [43]). We state our first biological law.

BL1. Stochastic Complexity Law. Physical processes, configurations and interactions at the atomic scale proceed stochastically towards greater complexity subject to natural laws, already attained structures and other boundary conditions.

High kinetic energy limits formation of complex physical systems since it rips clusters apart. Moreover, the more complex the configuration or process the less likely it occurs. Living organisms emerged only when the Earth cooled sufficiently that allowed more complex clustering by carbon atoms. Carbon has four valence electrons that bond with other carbon atoms to form huge strong organic compounds, e.g., the cells, tissues and organs of biological organisms. Carbon molecule has the strongest possible bonding owing to its simplicity, symmetry, stability and "rule of 8" principle of chemistry. In the hot early universe only simple prima formed. BL1 debunks the common belief that carbon, e.g., oil and coal, comes from decayed organisms which are more complex. Like crystals, carbon deposits do multiply. They also form in the atmosphere.

BL2. Stability Law. Although all possible configurations and clustering of matter may arise stochastically only stable ones remain or are replicated; this is the basis of natural selection.

BL2 is a generalization of Darwin's theory of natural selection. Physical systems that violate a physical principle are unstable and vanish in due course. Examples are man-made short-lived prima (by non-redundancy and non-extravagance principles). In biology unstable species become extinct.

The overwhelming base element of all living things on Earth is carbon although there are rare known metal-based living things underground [3] [44].

Since heat agitates and keeps prima simple it raises the chance of formation of more complex configurations of matter. This accounts for the considerable diversity of living organisms coming from hot magma oozing out of cavities in the Earth's interior, e.g., calderas and volcanic Galapagos and Hawaiian Islands.

In his Dialectics of Nature [32] Friedrich Engels has the natural law, Quantity-Quality Transformation Law which we modify as follows.

BL3. Existence of Nodal Points. Development including physical processes and evolution while generally smooth over a long period of time may be interrupted by abrupt changes or jumps called nodal points.

Examples of nodal points in nature are: $0^{\circ} \mathrm{C}$ (water converts to ice or ice to water), $100^{\circ} \mathrm{C}$ (water converts to steam or steam to water), emergence of snake species with toxin (bifurcation), and life from non-life with the formation of the gene. BL3 contradicts Darwin's theory of evolution directly [45]. In each case the quantization principle holds.

Engel's Quantity-Quality Transformation Law says, essentially, that a nodal point is the result of small or quantitative changes. This is not true with respect to the issue of the "missing link", i.e., the existence of species 
that lies between the primate crawling on four legs and the erect human species standing on two. According to Darwin changes in species are smooth and infinitesimal becoming noticeable over time. Thus, Darwin's theory says that there is an intermediate species between the crawling primates and the erect humans. Engels' dialectics does not rule it out either; he simply says that such nodal points exist but not necessarily where and when. If such a creature exists it must stand on two feet with elongated body slanted away from the vertical and attached to the legs at lower end. This posture is energy dissipating since the leg joints would be battling against the torque exerted by its weight due to gravity. Such species would be unstable and become extinct quickly (Stability Law) if it existed.

BL4. Opposite Forces (Tendencies) Resolution Law. Motion or process proceeds through synchronized resolution of opposite forces or tendencies.

Water wave, for instance, is synchronized vibration of the medium (true of all waves, e.g., electromagnetic waves), due to synchronized resolution of two opposite forces-water pressure and gravity. Water wave is generated at a point on the surface, say, by dropping a piece of rock on it that pushes the water molecules down the surface. Water pressure pushes them up above the surface due to momentum. (Pressure is effect of Brownian motion of the molecules due to the impact of cosmic waves) Gravity pulls them down again below the surface due to momentum, etc., resulting in vibration of the water molecules in place. The vibration resonates with surrounding molecules propagating it radially along concentric circles in accordance with the following natural law discovered in the course of analyzing and explaining the disastrous final flight of the Columbia Space Shuttle in 2004 [4] [5].

BL5. Resonance Law. Maximum resonance between waves or vibration occurs when they have the same characteristics including wave-length as well as variation of amplitude. The degree of resonance declines as the difference between wave characteristics and orders of magnitude of wavelengths rises, the latter principal. However, at suitably high order of magnitude of wavelength the infinitesimal effect of resonance with orders of magnitude nearby becomes considerable.

Example: when a tuning fork vibrates, another tuning fork of same frequency wavelength vibrates. The next law is an extension of BL5 and has medical applications [1] [6].

BL6. Law of Resonance and Superposition. Two waves of same order of magnitude resonate maximally when they have exactly the same characteristics and the principal determinant of level of resonance is wavelength or frequency; when two waves of different characteristics resonate their wave characteristics superpose on each other and form composite wave; in the case of a wave and a physical system resonance occurs when the wavelength of the former is comparable to the wavelength of the natural vibration of the latter or its measure across.

\subsection{The Gene}

The gene is the first physical system formed in nature that when adjoined to a protein strand (virus) exhibits properties of living cell. The gene consists of a sequence of four nucleotide bases, adenine paired with thymine through hydrogen bonding and guanine paired with cytosine also joined by hydrogen. In an organism the genes form a sequence in the cellular nucleus that makes up each of the pair of DNA parallel helical strands of the same composition that are mirror images of each other coiled into a helix in the chromosomes (the topologically appropriate configuration for passing on the genes to daughter cells in mitosis without entanglement). Genes of living cell, particularly, their sequencing of base elements, determine its physical characteristics and behavior; for a disease, this extends to its symptoms. This is the content of the next biological law (modified), a generalization of Mendel's genetics and contribution to the theory of evolution [46].

A major milestone in biology is the discovery that the common medium of the brain and gene for their functions are brain waves. Brain waves are electromagnetic waves encoded with the vibration characteristics of the neurons the principal determinant of which is the natural variation of the genes encoded on it. The primary function of the brain is control and coordination of body functions, processes and conscious motion directly through the creative-integrative region (CIR) [15] [16] and automatic functions through the secondary nervous system centered in the spinal column and extending through the network of nerves down the extremities but monitored by the CIR. Its secondary function is thought [15] [16]. That the brain's medium for its functions are brain waves follows from the finding in [47] [48] that pain sensation (due to vibration of neural chains by brain waves [15] [16] which occurs in the CIR produces molecule in the neural cellular membrane and the principles of uniformity, non-redundancy and non-extravagance. The primary function of the gene is production of tissues of living things through the brain waves it emits that convert superstrings to prima in the cellular membrane its 
secondary function passing on the genetic code to the offspring cell in mitosis. Substances in the human body are produced through the same conversion of superstrings to prima in the glands and cellular membranes but sometimes indirectly and in rare cases by thought (e.g., birthmark) [3]. That the gene's medium for its functions are brain waves follows from the same finding in [47] [48] and the principles of uniformity, non-redundancy and non-extravagance. The gene does its primary function by emitting its brain waves and agitating the superstrings in the cellular membrane and converting them to prima that make up the atoms and molecules of the tissues of a living thing its secondary function passing on the genetic code to the offspring in reproduction. Consider this example. Thought emits brain waves [16] [17] and when one sees or thinks of a lemon, brain waves vibrate the gene in the salivary gland via resonance and produces saliva. This is an example of automatic secretion as most secretions are; reflex is also a genetic automatic function. (Inside the cell, the 37 genes in the mitochondria produce the energy and substances that support it and its functions.) It is the fractal nature of brain waves generated by the nuclear vibration of the gene (made up of fractal superstrings) that endows them huge energy and the gene the power to convert dark to visible matter that determines its structure, properties and behavior through its vibration characteristics and sequencing of its base materials. This is the basis of the next biological law, a generalization of Mendel's genetics [46].

BL7. The Determinant Law. Each gene determines a specific physical characteristic of a plant or animal; conversely, every physical characteristic of a plant or animal is determined by a gene.

The gene distinguishes living from nonliving thing, the virus being the simplest. It is not an organism and cannot reproduce by itself. Therefore, it enters the nucleus of a host cell, emits its brain waves that agitates and converts superstrings in the cellular membrane of the host cell to prima that form the same virus in accordance with BL7. Since electromagnetic wave is fractal (being generated by the normal vibration of the fractal atomic nucleus) it is highly energetic. In the human body brain waves emitted by the genes agitate and convert superstrings to prima that form the tissues in the cellular membrane. The gene is replicated in every cell of the body.

How does nature insure that every part of the body grow in the right place? A gene turns on only in the right place to convert superstrings to suitable molecules on cue from the surrounding environment. This is how the cells and tissues are produced in various parts of the body which are determined by the genes responsible for their development. The specific environment is determined by enzymes and distribution of cumulus cells that initially surround the egg cell. Some chemicals are produced by specialized glands in the same way. Moreover, even turn-over of cells is genetic in the sense that each replacement has the genetic code of the oxidized old cell. We articulate our findings.

BL8. Law of Genetic Activation. Activation of a gene proceeds on cue from the immediate environment; this avoids anomaly in the development of an organism.

The next law sheds light on genetic alteration, mutation or alteration and evolution.

BL9. Genetic Brain Wave Propagation and Encoding. In an organism the gene generates and propagates brain waves; it is in harmony and at resonance with all its genes. A foreign gene or mutant may have discordant resonance with the chromosome that agitates the superstrings there and converts them into its mirror image in one of the pair of DNA strands of the recipient cell. Then it quickly produces its mirror image on the other DNA strand of the pair yielding a new gene, a mutant that determines new characteristics.

Mutation is complete when every cell has been similarly altered genetically. Then this characteristic becomes hereditary. First, a gene is altered then other genes also, locally at first, until it spreads to the entire body. It follows that genetic disorder may be acquired but once acquired it gets encoded in other cells and may not be removed beyond a certain period. It can only be neutralized by a neutralizer gene or eliminated without side effect by electromagnetic treatment [1] [3] [6] [13].

BL9 is the basis of the phenomenon of extra sensory perception (ESP) where one's brain waves resonates with someone else's far away. There is significant scientific verification of it in dogs reported by Science and Scientific American.

Corollary. A foreign gene may, by resonance, superpose its brain waves on brain waves emitted by a host organism to form composite brain waves that modify that gene accordingly (has applications to medicine in [1] [6] [13]).

In the application of the Internal-External Factor Dichotomy Law to evolution the gene is the internal and principal factor and the environment the external and secondary factor, a departure from Darwin.

A cell is altered when suitable new gene is introduced their brain waves superposing on each other and forming composite vibration characteristics. In effect, their base elements form a composite gene. Genetic alteration 
also involves changes in the sequence of genetic base elements. Radiation including background radiation may also cause genetic alteration; so does direct exposure to a foreign gene, especially, a primate's. Frequent, consistent and sustained use of body part may create a new gene in accordance with the Determinant Law. It is known that like the gene, the neuron emits energetic brain waves that produce molecules in its cellular membrane [47] [48] or alters a gene in the chromosome. The latter case is the basis of some birthmarks. For example, during conception a pregnant woman may crave for or be pre-occupied with some object, living or not. Then the baby may have birthmark resembling the object [3] [6]. Genetic alteration has medical applications. In the treatment of cystic fibrosis, for instance a lever virus, e.g., cold virus, is infected with a neutralizer gene for cystic fibrosis and inhaled. Its brain waves modify the cystic fibrosis gene and removes the undesirable symptoms. We summarize our findings.

BL10. Law of Genetic Alteration. The possible sources of genetic alteration are: a) radiation, e.g., electromagnetic waves, $b$ ) exposure to foreign gene, $c$ ) frequent, consistent and sustained use of body part, $d$ ) exposure to some chemicals, e.g., PCB, e) pre-occupation with some object and $(f)$ introduction of neutralizer gene.

This law has application to training of athletes (drills) by creating suitable reflexes and strong muscles both of which are genetic. It applies to training of performing artists, e.g., pianist. It is also manifested in the evolution of species that walked on four legs and became erect. Occasionally, members stood up to see farther in search of food or spot intruder. Over time, this new physical characteristic of standing erect became encoded genetically along with appropriate reflexes and features until standing became a natural posture. Then the front legs found new functions and the anatomy of both front legs and the face were genetically encoded and modified accordingly.

Rice farmers on plain fields in rural Philippines develop flat feet by sustained and consistent walking on mud, sometimes waist-deep (flat foot sinks less on mud). The effect is thickening of the tissues of the flat of the foot which is genetically encoded. While flat-footedness is subject to Stochastic Complexity this physical feature is the stable one in this situation. Farmers also develop oversized protruding strong big toes that are slightly arched away from the little toes by sticking their big toes into the slippery soil while climbing steep hills. This is reinforced by climbing coconut trees by sticking the big toes on narrow cuts on the trunk. The Stochastic Complexity and Stability Laws along with BL10 account for it. Genetic alteration in an adopted child follows from the corollary.

A person in pain may cope with it by adjusting posture, e.g., when the pain is in the spinal column, he adjusts posture to ease it and when sustained suitably corresponding genetic alteration and encoding occur.

It is popularly believed that humans originated in Africa and that humans elsewhere in the globe are the result of migration. This is at odds with the Stochastic Complexity and Stability Laws since all species come from the same stuff-superstrings - that fill up the entire Cosmos. It follows that the existence of life (even intelligent beings) elsewhere in the Cosmos is not remote.

The next law offers further explanation of mutation and birthmarks.

BL11. Law of Induced Mutation, Genetic Alteration and modification. When a new gene has discordant resonance with a gene in the host cell its brain waves agitate its chromosomes and produce its mirror image there in one DNA strand that replicates itself in the opposite strand to form a mature gene. When brain waves of foreign gene resonate with a gene of the host cell the characteristics of one superpose on the other and produce a composite (modified) gene that emits its composite brain wave characteristics.

\subsection{Evolution, Control and Advancement}

Reproduction insures continuance of a species (survival) subject to the environment which may change over time and affect its course in accordance with the Stability Law. A series of mutations changes the genetic code of species leading to a) quantitative improvement or b) nodal point where a new species splits off from the old (bifurcation). However, nodal point in evolution is not always the result of quantitative advancement but Energy Conservation and Stochastic Complexity Laws and quantization principle. Control refers to dominance over the environment and other species and advancement means rising capability to cope with problems of survival and control including improvement of intelligence [3] [6].

\subsection{Reproduction}

From the simplest to the most complex living thing beyond virus, reproduction is, essentially, cell division. The 
only new element is fertilization in plants and animals but even this is followed by cell division that results in the growth of the embryo.

We do not repeat what is known about reproduction but highlight what is new and relevant about it.

1) The gene sequencing is part of the program for reproduction that determines the physiology and configuration of the molecules that form the tissues and organs of the organism. The genes in the DNA of an organism, arranged in sequence of base materials that form them, encode its entire structure, physiology, physical characteristics, instincts and reflexes.

2) The only topologically feasible DNA symmetric replication without entanglement during mitosis is for the double helix to split longitudinally along it between the two pairs of DNA strands, half of the pair slightly contracting as inner coil and sliding out into one of the offspring cells. Then in each offspring cell a DNA strand replicates its mirror image in the parallel chromosome through the brain waves it emits to complete its parallel pair of DNA strands, again, a matured DNA pair. Male or female, the mirror image of the genes in the offspring cell is in the $\mathrm{x}$-chromosomes since if the $\mathrm{x}$-chromosomes from the male go to the offspring cell its DNA there will form its mirror image in the $\mathrm{x}$-chromosomes of the female (to produce a female offspring with $\mathrm{x}$-, $\mathrm{x}$-chromosomes); so does the DNA in the y-chromosomes of the female offspring.

3) The egg has half the x-chromosomes of the female containing one of the pair of DNA strands. Each of the male's sperm cells has either the x-chromosome with its DNA strand or the y-chromosome with the other DNA strand of the pair. Therefore, it has half the chromosomes.

4) Fertilization occurs when the sperm cell penetrates the egg cell and inserts its $x$ - or $y$-chromosomes to pair with the latter's helical DNA strand and form parallel pair of helical DNA strands. The process of pairing is the reverse of mitosis, the helical half-chromosomes of the sperm inserting itself into the helical half-chromosomes of the egg, expands slightly to form the parallel pair of the female helical DNA the result of which is a cell containing the pair of parallel helical DNA strands in the $\mathrm{x}-$, $\mathrm{x}$ - or $\mathrm{x}-$, $\mathrm{y}$-chromosomes. By resonance, a gene from each partner that will produce the tissues of the same part of the body of the offspring forms a pair, one on each DNA strand of the helical pair of the fertilized egg. Their brain waves superpose on each other to form composite wave characteristics and modify each other accordingly to emit the same composite wave characteristics. The modified gene pair determines the characteristics of that offspring's body part. If the brain-wave-characteristics of a partner's gene are more prominent than those of its pair this partner's physical characteristics will be dominant in this offspring, recessive otherwise.

5) A gene of one partner that does not have a counterpart in the other produces its mirror image on the DNA strand contributed by the other partner so that it is shared by the offspring. One partner's gene is dominant over the other partner's if its emitted brain wave characteristics are more prominent in the composite brain wave. The component physical characteristics determined by a gene are present in each partner only that one is more prominent in one than in the other.

6) The male genital has counterpart components in the female genital the difference being that the male characteristics are dominant in the former (and vice versa), differentiation reinforced by male hormones.

7) In rare cases (e.g., hermaphrodite) there is no clear dominance of one gender characteristics over the other so that the individual has both penis and vagina. Dominance is sustained and expressed in feelings, emotions and personality make-up by the appropriate hormones (genetically determined) but hormonal imbalance can erode them. Moreover, the levels of hormones and enzymes and nature of physiological process involved in the development of the fetus are genetically determined. However, since genetic alteration is formed in accordance with Stochastic Complexity and regulated by the environment through the Stability Law "gayness" may appear at any point in the family tree (applicable to both gay men and women).

8) Fertilization quickly triggers formation of gene mirror images, composite genetic brain waves and corresponding genetic modification. Since it involves release of energy as brain waves it de-stabilizes the fertilized egg that is resolved by mitosis. Mitosis conforms to energy conservation in terms of symmetry of end result, namely, the replication or fractalization of cells with shared structure. We state our findings as a biological law.

BL12. Law of Reproduction and Correspondence. During fertilization the single full DNA strands from the sperm and egg are paired, by Resonance, so that 1) each pair of genes that produces the same part of the embryo are opposite each other in the chromosomes of the fertilized egg; 2) their brain waves superpose on each other resulting in common composite brain waves; 3) unpaired genes from each parent DNA produce their mirror images in the other to complete genetic composition of the fertilized egg and 4) the energy involved in (1)-(3) causes instability in the fertilized egg that triggers mitosis and starts the development of the embryonic 
offspring.

In humans the embryonic cells are physically identical during the first few days of cell division. Differentiation begins with changes in nuclear instruction in the activation of the genes by turning them on, i.e., translating genetic codes to produce appropriate tissues and chemicals or keeping them turned off temporarily for some and permanently for others. For example, the genes that will produce the tissues of the embryonic heart will turn on and produce the right molecules in the cellular membranes there including the base molecules of genetic materials where the genes will be replicated along with the cell division that builds the embryonic heart. The genes there with genetic instruction to produce the pancreatic tissues are permanently shut off and eventually excreted when the cell dies.

During the period of growth of an individual the cells multiply exponentially and die linearly (mainly through oxidation). When the individual reaches full growth the growth function tapers off to a linear function. At old age the linear growth function takes a lesser slope than the cell death function, the individual shrinks and functioning neural cells decline. However, there is therapy for countering the effect of ageing at least, mentally. One is to keep active by doing creative work both mentally and physically since the brain is in charge of all such processes [15] [16]. The effect is to activate new neural chains (connectors) and network that offsets the narrowing of the brain and delay the onset of dementia and Alzheimer.

The present human species took millions of years to achieve by the combination of Stochastic Complexity, Stability and Energy Conservation Laws. Trillions of different structures emerge from this fractal process, ineffective and unstable ones discarded and replaced by stable ones, until present structures and level of precision are achieved which, even now, are not perfect yet as genetic diseases still exist.

The reproductive process has practical applications in agriculture, especially, plant breeding. In medicine scientists are able to isolate a defective gene; then a neutralizer gene is injected into the patient to alter the genetic code and remove the undesirable symptom and cure the ailment. Now molecular biology and genetics can engineer stem cells that can repair dead tissues and offset cell degeneration due to aging.

\subsection{Central Role of the Gene in Evolution}

The Determinant Law is the key that pushes evolution forward. In the development of a species new genes appear steadily in accordance with Stochastic Complexity. If the specific characteristics determined by the genes fall within the parameters favored by the environment with respect to survival, control and advancement, the recipient species will remain stable or even advance; otherwise, it becomes extinct. Needless to say, changes in the environment alter the stability of species. If new genes create new species then bifurcation occurs and a new species of different genetic content emerges and departs from the old one. Otherwise, there is simply continuation or advancement. For example, six million years ago, the snake had a pair of legs located a third of its length from the tail's tip. That must have been clumsy and unstable. Bifurcation occurred, the new species acquiring agile body that made it wiggle through the bushes and climb trees with ease. Then the pair of legs atrophied but left a pair of scars. Thus, there is some gene that encodes them, perhaps, an alteration of the original.

A nodal point occurred that gave rise to huge muscular snake, e.g., python, anaconda, the old species remaining. Then size became a disadvantage (instability): a) it requires huge amount of food to sustain and b) their prey that had also become smaller could hide in holes or crevices for their survival; these aside from competition for food with other snakes. At this point bifurcation occurred resulting in appearance of smaller snakes that catch their prey in holes or crevices. Then another bifurcation occurred: appearance of snakes with venom for protection, digestion.

For complex living thing it takes a series of mutations to reach a nodal point in accordance with the QuantityQuality Transformation Law. However, for the virus it takes only a single gene mutation to create a new virus. That is why virus in other species strikes fear in humans that it can mutate and transfer to them. Some microbes mutate and turn the cure into food for them, e.g., some species of typhoid and malaria [3] [6].

Such changes and advancement are in accordance with BL1 and BL2.

\section{Electromagnetic Treatment of Infectious Diseases}

An extension of [1], this paper applies GUT and UTE to the treatment of infectious diseases. It is a type of GUT technology discussed in [17]. 


\subsection{Important Relevant Results}

We highlight relevant results from [1] [3] [6] [13] [15] for our purposes.

1) Brain waves are the medium of both the brain and the gene for their functions [3] [6]. The brain's main function is control of body movements, processes and physiology; automatic motion and processes are delegated to and controlled by the secondary nervous system centered in the spinal column and extending through the entire network of nerves but monitored by the CIR. For example, when the finger touches hot iron the automatic nervous system pulls it first and reports to and registers in the creative-integrative region (CIR) [3] [6] for interpretation and appropriate action; thus, sensation is delayed.

2) All body processes, responses and reactions are monitored by the CIR for interpretation and appropriate response [3] [6] [13]-[16] Sometimes automatic action comes from the brain [3] [16]. For example, thinking about lemon triggers automatic salivary secretion, i.e., dark to visible matter conversion by brain waves in the salivary glands. The main function of the gene is to produce living tissues in the cellular membrane, its secondary function to pass itself on to the offspring in reproduction. In the former, the gene emits radiation (brain waves) that converts superstrings to prima in the cellular membrane in accordance with the Genetic Activation Law (more in [3] [6].

3) A new gene (mutant) in the body emits brain waves in all directions that convert superstrings in one of the pair of chromosomes of each cell to basic prima that form its mirror image as sequence of base elements, a new gene. This new gene emits brain waves that produce its mirror image in the opposite chromosome to form a new pair of mature genes. When the DNA strand splits during mitosis each gene in a pair is passed on to the daughter cell, creates its mirror image and form a new pair of matured genes and the process and DNA are replicated there. A gene from another species may similarly project its brain waves, form its mirror image in the human chromosome and create a new gene. This is the scientific basis of birthmark. Birthmark may also be self-induced on the embryo of the pregnant mother by being mentally pre-occupied with an object, living or not, projecting brain waves that create the gene encoded with its physical characteristics [3] [6] [13]-[16].

4) Over time, all cells will be endowed with this new gene but it will produce tissues and chemicals selectively in accordance with the Genetic Activation Law where every cell produced will carry this gene.

5) Not all genes are inherited from the parents; some are acquired. Once acquired, a gene replicates itself in every cell over time and can be removed only by genetic sterilization since the sterilized gene is not retained in the new cells in the normal turnover of cells and eventually excreted with the dead cells.

6) In cystic fibrosis a neutralizer gene is introduced in the lung through a lever virus infected with it that enters the nucleus of the cell. The neutralizer gene recreates itself in the chromosome objectively producing a composite with the cystic fibrosis gene in the lung without the undesirable symptom (e.g., clogging the lung with mucous) while at the same time recreating itself in the chromosomes of the other cells with the same effect. Not only does it control the disease, it treats it.

7) The treatment in 6) can be applied to other genetic diseases by introducing the appropriate neutralizer genes for specific genetic diseases.

8) By identifying the vibration characteristics of the natural vibration of the defective gene (of the disease) which is defined primarily by its size, i.e., measure across as sequence of base materials and segment of a double helix, radiation with the same vibration characteristics may be generated to resonate with the defective gene alone (analogous to selective resonance in the microwave oven that heats up food alone). Then the patient can be exposed to it and by raising its intensity sufficiently it will sterilize the defective gene, i.e. stop the spread of the disease and render it harmless to the patient without harm to normal cells. It will not replicate itself in the normal turnover of cells and the patient will be cured without side effect. Since genetic vibration is a composite of the vibration of its base elements, it may be sufficient to sterilize a base element alone and remove the brain waves responsible for the undesirable symptom.

9) In the case of a virus another target for resonance is its protein strand (with its gene). The appropriate radiation has wavelength comparable with its size (measure across). If there is no system in the body comparable with it in size (which is likely since the virus is much smaller than the cells) then the radiation will burn the virus without injury to normal cells.

\subsection{Extending Electromagnetic Treatment to Infectious Diseases}

The treatment referred to in (6) and (7) applies only to genetic diseases (symptoms determined by their genes) 
such as cancer, systemic lupos erythematosus, diabetes, muscular dystrophy and mental disorder which are neither infectious nor contagious. It does not apply to infectious diseases including viruses, e.g., ebola, which reproduces and spreads quickly. However, we can apply (8), a special case of electromagnetic treatment, that utilizes the Resonance and Genetic Sterilization Laws [3] [6]. This will entail identification of the natural vibration wavelength or the size of the determinant gene of the disease and if the wavelength does not distinguish it from those of the genes of the host (patient) another distinguishing characteristic is added: amplitude variation so that radiation can be produced that would resonate with that gene alone. Raising the intensity of the radiation suitably to sterilize the disease will stop it from spreading to other patient and render it harmless to the patient. In the case of the virus we can apply (9) by targeting the size of its protein strand (with the gene) for resonance by knowing its size. Certainly, the virus size is smaller than the normal cells and burning it with the resonant radiation would not harm them. These treatments have no side effect. They are the obvious ones at this time. However, specialists in infectious diseases may be able to find other distinguishing characteristics that can be targeted for resonance towards treatment without side effect.

If the wavelength of the natural variation of the gene of the virus does not distinguish it from the normal genes for purposes of resonance its amplitude variation must be added to the radiation characteristics of the appropriate radiation for treatment without side effect. This applies also to the radiation resonance with the virus itself for treatment. The amplitude variation of the gene is superposed on the natural variation of the virus.

For infectious diseases in general, the same treatment via genetic resonance applies but requires identifying the genes responsible for the undesirable symptoms and sterilizing them. The infectious disease is genetically altered [6] and not sterilized but rendered harmless.

\section{Collaborative Research and Development}

Collaborative and research and development towards the design and fabrication of appropriate device for genetic sterilization of each of the infectious diseases (e.g., fungus that infects the prostate) will require participation of experts in GUT, UTE, the genetic disease concerned who can identify the vibration characteristics of the determinant gene, especially, wavelength and amplitude variation, a nuclear engineer who can identify suitable radioactive material to provide the required resonant radiation, if any, and radiation engineer who can design and fabricate such devise to emit the desired resonant radiation. If the wavelength is already sufficient to distinguish the defective cell (or gene) from normal cell (or gene), then the variation characteristics can be ignored. Only the determinant gene responsible for the undesirable symptom of the disease needs to be sterilized or disabled to treat the disease. The central natural laws involved in this treatment are the Resonance and Internal-External Dichotomy Laws where the latter specifies the signature vibration characteristics of every physical system.

\section{References}

[1] Escultura, E.E. (2012) Electromagnetic Treatment of Genetic Diseases. Journal of Biomaterials and Nanobiotechnology, 3, 292-300. http://dx.doi.org/10.4236/jbnb.2012.322036

[2] Escultura, E.E. (2008) The Grand Unified Theory. Nonlinear Analysis, A-Series: TMA, 69, 823-831. http://dx.doi.org/10.1016/j.na.2008.02.043

[3] Escultura, E.E. (2015) The Unified Theory of Evolution. The Journal of the Science of Healing Outcomes, in Press.

[4] Escultura, E.E. (2007) The Pillars of the New Physics and Some Updates. Nonlinear Studies, 14, 241-260.

[5] Escultura, E.E. (2013) Columbia’s Disastrous Final Flight. In: Escultura, E.E., Ed., QMAM: TPA, LAP LAMBERT Academic Publishing, Saarbrücken, 281-293.

http://www.barnesandnoble.com/w/qualitative-mathematics-and-modeling-escultura-edgar/1117374460?ean=9783659 $\underline{305849}$

[6] Escultura, E.E. (2011) Genetic Alteration, Modification and Sterilization with Applications to Treatment of Genetic Diseases. The Journal of the Science of Healing Outcomes, 7, 2015.

[7] Escultura, E.E. (1970) The Trajectories, Reachable Set, Minimal Levels and Chains of Trajectories of a Control System. Ph.D. Thesis, University of Wisconsin.

[8] (2014) The Leap from Traditional to the New Science. Nonlinear Studies, 21, 283-291. http://nonlinearstudies.com/index.php/nonlinear/article/view/1001

[9] Escultura, E.E. (1997) The Solution of the Gravitational n-Body Problem. Journal of Nonlinear Analysis, A-Series: Theory, Methods and Applications, 30, 5021-5032. http://dx.doi.org/10.1016/S0362-546X(96)00133-2 
[10] Escultura, E.E. (2013) The Logic and Fundamental Concepts of the Grand Unified Theory. Journal of Modern Physics, 4, 213-222. http://dx.doi.org/10.4236/jmp.2013.48A021

[11] Escultura, E.E. (1998) Exact Solutions of Fermat's Equation (A Definitive Resolution of Fermat's Last Theorem). Journal of Nonlinear Studies, 5, 227-254.

[12] Escultura, E.E. (2009) The Mathematics of the Grand Unified Theory. Nonlinear Analysis, A-Series: TMA, 71, e420e431.

[13] Escultura, E.E. (2010) The Origin and Evolution of Biological Species. The Journal of the Science of Healing Outcomes, 17-27.

[14] Escultura, E.E. (2013) The Theory of Intelligence. In: Escultura, E.E., Ed., Qualitative Mathematics and Modeling: Theoretical and Practical Applications, (QMAM: TPA), LAP LAMBERT Academic Publishing, Saarbrücken, 187-212. http://www.barnesandnoble.com/w/qualitative-mathem-ma-tics-and-modeling-escultura-edgar/11173744460?ean=9783 $\underline{659305849}$

[15] Escultura, E.E. (2012) The Physics of Intelligence. Journal of Education and Learning, 1, 51-64. http://www.scirp.org/Journal/Home.aspx?IssueID=1673 http://dx.doi.org/10.5539/jel.v1n2p51

[16] Escultura, E.E. (2014) The Physics of Physics of the Mind. The Journal of the Science of Healing Outcomes, 6, 6-15.

[17] Escultura, E.E. (2013) GUT Technology. In: Escultura, E.E., Ed., QMAM: TPA, LAP LAMBERT Academic Publishing, Saarbrücken, 294-335.

http://www.barnesandnoble.com/w/qualitative-mathemmatics-and-modeling-escultura-edgar/1117374460?ean=978365 9305849

[18] Escultura, E.E. (2013) Practical Applications of the Grand Unified Theory. Asian Online Journal. www.asianonlinejournals.com

[19] Escultura, E.E. (2014) Is Human Activity Linked to Climate Change? Atmospheric and Climate Sciences, 4, $305-316$. http://file.scirp.org/Html/15-4700251_45173.htm http://www.scirp.org/journal/acs

[20] Escultura, E.E. (2013) Problems of Modelling in Geology and Oceanography. Problems of Nonlinear Analysis in Engineering Systems (IFNA-Russian Academy of Sciences), 19, 164-178. http://kpfu.ru/portal/docs/F2118903903/Zurnal40.pdf

[21] Escultura, E.E. (2001) Turbulence: Theory, Verification and Applications. Journal of Nonlinear Analysis, A-Series: TMA, 47, 5955-5966.

[22] Escultura, E.E. (2013) Chaos, Turbulence and Fractal: Theory and Applications. International Journal of Modern Nonlinear Theory, 2, 176-185. www.scirp.org/journal/ijmnta http://dx.doi.org/10.4236/ijmnta.2013.23025

[23] Escultura, E.E. (2012) Creative Mathematics Education. Creative Education, 3, 45-54. http://www.scirp.org/journal/Home.aspx?IssueID=1404 http://dx.doi.org/10.4236/ce.2012.31008

[24] Escultura, E.E. (2013) Creative Science Education. In: Escultura, E.E., Ed., QMAM: TPA, LAP LAMBERT Academic Publishing, Saarbrücken, 306-320.

http://www.barnesandnoble.com/w/qualitative-mathem-matics-and-modeling-escultura-edgar/1117374460?ean=97836 $\underline{59305849}$

[25] Escultura, E.E. (2013) Strategic Positioning towards Sustainable Development of the Third World. In: Escultura, E.E., Ed., QMAM: TPA, LAP LAMBERT Academic Publishing, Saarbrücken, 322-337.

http://www.barnesandnoble.com/w/qualitative-mathem-matics-and-modeling-escultura-edgar/11173744460?ean=97836 59305849

[26] Sarma, V.S.R.S. and Escultura, E.E. (2010) Proceedings of International Conference on Sustainable Development, Excel India Publishers, Chennai, 1-6 February 2010, 241-243.

[27] Astronomy, August 1995.

[28] Watson, A. (1998) Glow Reveals Early Star Nurseries. Science, 17, 332-333.

[29] Glanz, J. (1998) Starbirth, Gamma Blast Hint at Active Early Universe. Science, 282, 1806.

[30] Escultura, E.E. (2011) Extended Geometrical and Generalized Fractals, Chaos and Applications. In: Brennan, K.J., Ed., Handbook on the Classification and Application of Fractals, Nova Publishers, Hauppauge, 1-39. https://www.novapublishers.com/catalog/product_info.php?products_id=23231

[31] Edgar, G.A. (1990) Measure, Topology and Fractal Geometry. Springer-Verlag, New York. http://dx.doi.org/10.1007/978-1-4757-4134-6 
[32] Haldane, J.B.S. (1939) Engels’ Dialectics of Nature. http://www.marxists.org/archive/marx/works/1883/don/preface.htm

[33] Atsukovsky, V.A. (1990) General Ether-Dynamics; Simulation of the Matter Structures and Fields on the Basis of the Ideas about the Gas-Like Ether. Energoatomizdat, Moscow.

[34] Escultura, E.E. Macro Gravity. In: Escultura, E.E., Ed., QMAM: TPA, LAP LAMBERT Academic Publishing, Saarbrücken, 147-161.

http://www.barnesandnoble.com/w/qualitative-mathemmatics-and-modeling-escultura-edgar/11173744460?ean=978365 9305849

[35] Hellemans, A. (1999) The Mystery of the Migrating Galaxy Clusters. Science, 283, 625-626.

[36] (1995) Galactic Collision. Scientific American, 11-14.

[37] Gerlovin, I.L. (1990) The Foundations of United Theory of Interactions in a Substance. Energoattomizdat, Leningrad.

[38] Nieper, H.A. (1984) Revolution in Technology, Medicine and Society. Management Interessengemeinschaft für Tachyon-Feld-Energie, Oldenburg, FRG.

[39] Escultura, E.E. (2009) Qualitative Model of the Atom, Its Components and Origin in the Early Universe. Nonlinear Analysis, B-Series: Real World Applications, 11, 29-38. http://dx.doi.org/10.1016/j.nonrwa.2008.10.035

[40] Garisto, R. and Agarwal, A. (2012) The Import of the Higgs Boson. Scientific American, 307, 20-21. http://dx.doi.org/10.1038/scientificamerican0912-20a

[41] Escultura, E.E. (2011) Quantum Gravity. In: Escultura, E.E., Ed., Scientific Natural Philosophy, Bentham Science Publishers, Sharjah, 61-80. http://www.benthamscience.com/eBooks/9781608051786/index.htm

[42] Escultura, E.E. (2009) The New Real Number System and Discrete Computation and Calculus. Neural, Parallel and Scientific Computations, 17, 59-84.

[43] Escultura, E.E. (2013) Critique-Rectification of Mathematics. In: Escultura, E.E., Ed., QMAM: TPA, LAP LAMBERT Academic Publishing, Saarbrücken, 77-129.

http://www.barnesandnoble.com/w/qualitative-mathemmatics-and-modeling-escultura-edgar/1117374460?ean=978365 $\underline{9305849}$

[44] Discover, December, 1999.

[45] Darwin, C. and Quammen, D. (2011) The Origin of Species. Sterling, London.

[46] Mendelian Genetics. www.ndsu.edu/pubweb/ mcclean/plsc431/mendel/mendel1.htm

[47] Krylov, V.B. and Shehegelov, B.F. (1999) Mathematical Methods in the Physiology of Sensory Systems. Book of Abstracts, Proceedings of the Second International Conference on Tools for Mathematical Modeling, St. Petersburg, 14-19 June 1999, 14-19.

[48] Osipenko, G.S., Pokrovsky, A.N., Krylov, B.V. and Plakhova, V.B. (2000) Mathematical Modeling of Pain Sensation. Proceedings of the Second International Conference on Mathematical Modeling, St. Petersburg. 